\title{
Health-Enabling Technologies for Telerehabilitation of the Shoulder: A Feasibility and User Acceptance Study
}

\author{
Bianca Steiner ${ }^{1}$ Lena Elgert ${ }^{2}$ Birgit Saalfeld ${ }^{2}$ Jonas Schwartze ${ }^{2}$ Horst Peter Borrmann ${ }^{3}$ \\ Axel Kobelt-Pönicke ${ }^{4}$ Andreas Figlewicz ${ }^{5}$ Detlev Kasprowski ${ }^{5} \quad$ Michael Thiel $^{5} \quad$ Ralf Kreikebohm $^{6}$ \\ Reinhold Haux ${ }^{1}$ Klaus-Hendrik Wolf ${ }^{2}$
}

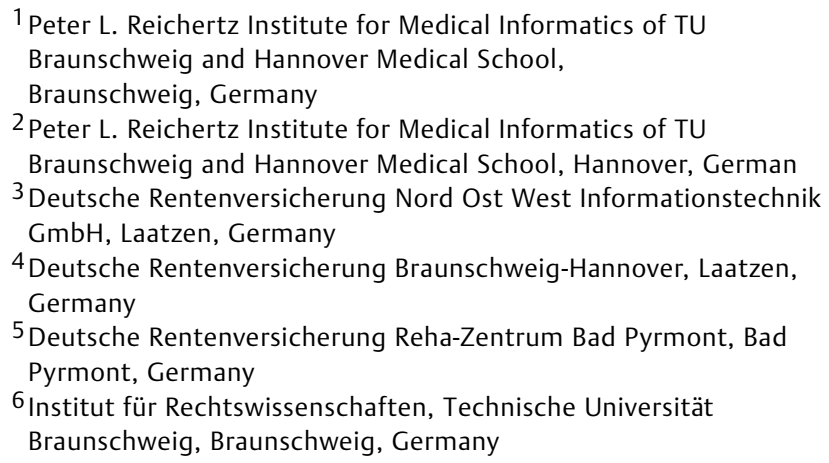

Address for correspondence Bianca Steiner, MSc, Peter L. Reichertz Institute for Medical Informatics of TU Braunschweig and Hannover Medical School, Braunschweig, Germany, Muehlenpfordtstr. 23, 38106 Braunschweig, Germany (e-mail: bianca.steiner@plri.de).

Methods Inf Med 2020;59:e90-e99.

\begin{abstract}
Keywords

- musculoskeletal diseases

- shoulder

- exercise therapy

- telerehabilitation

- evaluation studies

Background After discharge from a rehabilitation center the continuation of therapy is necessary to secure already achieved healing progress and sustain (re-)integration into working life. To this end, home-based exercise programs are frequently prescribed. However, many patients do not perform their exercises as frequently as prescribed or even with incorrect movements. The telerehabilitation system AGT-Reha was developed to support patients with shoulder diseases during their home-based aftercare rehabilitation.

Objectives The presented pilot study AGT-Reha-P2 evaluates the technical feasibility and user acceptance of the home-based telerehabilitation system AGT-Reha.

Methods A nonblinded, nonrandomized exploratory feasibility study was conducted over a 2-year period in patients' homes. Twelve patients completed a 3-month telerehabilitation exercise program with AGT-Reha. Primary outcome measures are the satisfying technical functionality and user acceptance assessed by technical parameters, structured interviews, and a four-dimensional questionnaire. Secondary endpoints are the medical rehabilitation success measured by the active range of motion and the shoulder function (pain and disability) assessed by employing the Neutral-0 Method and the standardized questionnaire "Shoulder Pain and Disability Index" (SPADI), respectively. To prepare an efficacy trial, various standardized questionnaires were included in the study to measure ability to work, capacity to work, and subjective prognosis of work capacity. The participants have been assessed at three measurement points: prebaseline (admission to rehabilitation center), baseline (discharge from rehabilitation center), and posttherapy.
\end{abstract}

received

October 30, 2019

accepted after revision

May 14, 2020 (c) 2020 Georg Thieme Verlag KG

Stuttgart · New York
DOI https://doi.org/

10.1055/s-0040-1713685.

ISSN 0026-1270.
License terms

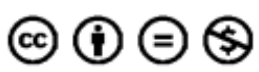


Results Six participants used the first version of AGT-Reha, while six other patients used an improved version. Despite minor technical problems, all participants successfully trained on their own with AGT-Reha at home. On average, participants trained at least once per day during their training period. Five of the 12 participants showed clinically relevant improvements of shoulder function (improved SPADI score $>11$ ). The work-related parameters suggested a positive impact. All participants would recommend the system, ten participants would likely reuse it, and seven participants would have wanted to continue their use after 3 months.

Conclusion The findings show that home-based training with AGT-Reha is feasible and well accepted. Outcomes of SPADI indicate the effectiveness of aftercare with AGTReha. A controlled clinical trial to test this hypothesis will be conducted with a larger number of participants.

\section{Introduction}

Musculoskeletal diseases (MSDs) are considered to be the leading worldwide cause of chronic pain, physical impairment, and loss of quality of life, which lead to high socioeconomic costs. ${ }^{1,2}$ Among them, shoulder lesions are ranked in the top 10 diagnoses responsible for the highest proportion of days of incapacity to work. ${ }^{3}$ The diagnosis of shoulder lesion contains different diseases of the shoulder joint, for example, shoulder impingement syndrome (SIS), frozen shoulder, and osteoarthritis of the shoulder. ${ }^{4}$ Apart from pain-reducing drugs, inpatient and full-day outpatient rehabilitative care are considered to be the standard therapy. ${ }^{5}$ The World Health Organization defines rehabilitation as "a set of measures that assist individuals, who experience, or are likely to experience, disability to achieve and maintain optimum functioning in interaction with their environments." ${ }^{6}$ Regarding employment, its general aim is to reduce unemployment and foster a durable (re-)integration into working life. ${ }^{5}$

Successful and sustainable rehabilitation requires a longterm provision of rehabilitation services even after discharge from a rehabilitation center. ${ }^{7}$ Self-reliant home-based exercise programs are one of the treatments prescribed to this end. ${ }^{6}$ However, many patients perform their physiotherapeutic exercises incorrectly or not as frequently as prescribed.

Prior experiences show an important contribution of health-enabling technologies to sustained rehabilitation success, especially when employed to support home-based exercises. In general, health-enabling technologies are defined as "sensor-based information and communication technologies, aiming at contributing to a person's health and health care as well as to his or her quality of life." 8

The majority of health-enabling technologies for rehabilitation focuses on patients with neurological disorders. This includes technologies currently in widespread use, health care service research activities, as well as future technologies under development. ${ }^{9-13}$ For patients with MSD of the shoulder, telerehabilitation services are in use to support homebased exercises by connecting patients and physical therapists. ${ }^{14}$ To the authors' knowledge, there are no home-based rehabilitation services to date that are supported by healthenabling technologies, include an automated real-time feed- back for patients with MSDs of the shoulder, and that have been evaluated for feasibility at patients' homes.

Beyond this background the telerehabilitation system AGT-Reha has been developed in close cooperation with physical therapists. It provides automated real-time feedback as well as time-delayed feedback from physical therapists. In contrast to traditional unsupervised home exercise programs, AGT-Reha enables patients to continue their rehabilitation exercises with teletherapeutic support.

The remainder of this article describes the feasibility evaluation study of AGT-Reha-P2. The system itself, as technical foundation of the intervention, is introduced briefly and set into context of related work. The aim and protocol of the study are described in the Objectives and Methods sections, followed by the Results, which were structured with regard to the main outcome measures.

\section{AGT-Reha}

AGT-Reha is a telerehabilitation system for patients with (chronic) shoulder diseases. It guides patients through physiotherapeutic exercises at home with automated real-time feedback. ${ }^{15}$ Measured exercise quantity and quality can be remotely observed by attending physical therapists.

Two major versions of AGT-Reha were used. The AGT-RehaV1 client setup consists of a Notebook with a 17-inch screen and the marker-less motion capture system (depth camera) Microsoft Kinect for Xbox 360 ( - Fig. 1, left). The "Kinect for Xbox 360" (Kinect v1) was originally developed for the game console Xbox 360. AGT-Reha-V2 uses the upgraded Kinect version "Kinect for Windows" (Kinect v2) with a refined client setup ( $=$ Fig. 1, right). The self-developed AGT-Reha client software includes a catalogue of nine physiotherapeutic exercises for the shoulder, partly with variants. The exercises were defined in cooperation with physical therapists of the rehabilitation center Bad Pyrmont, Germany. These exercises can be individually adapted for each patient during the whole homebased rehabilitation process via the AGTRehaCare Web interface. Physical therapists may use this to change the number of sets and repetitions per exercise, adjust the difficulty level, or modify the arrangement of exercises to avoid fatigue and boredom. The interface also allows physical therapists to examine the progress and training quality of each patient 

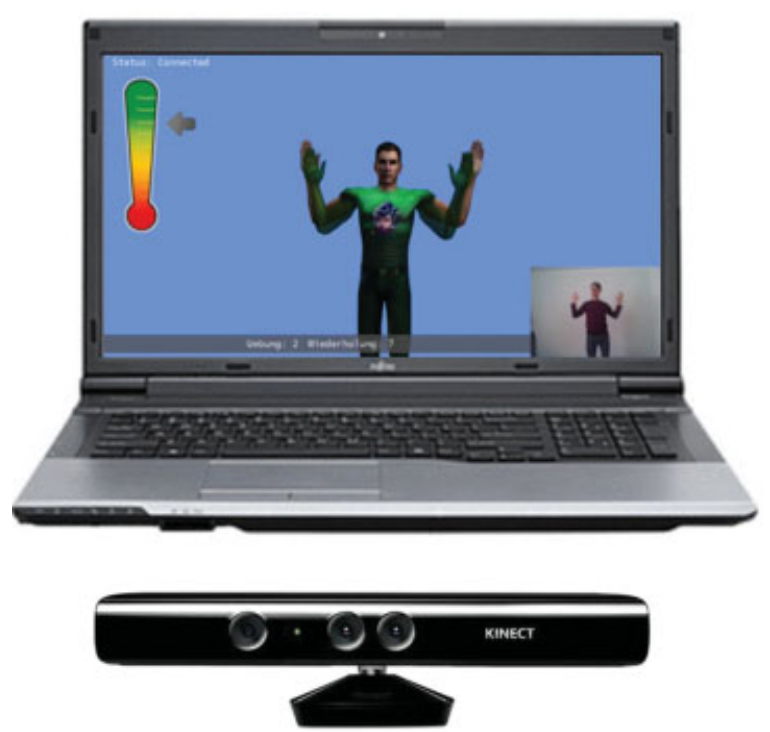
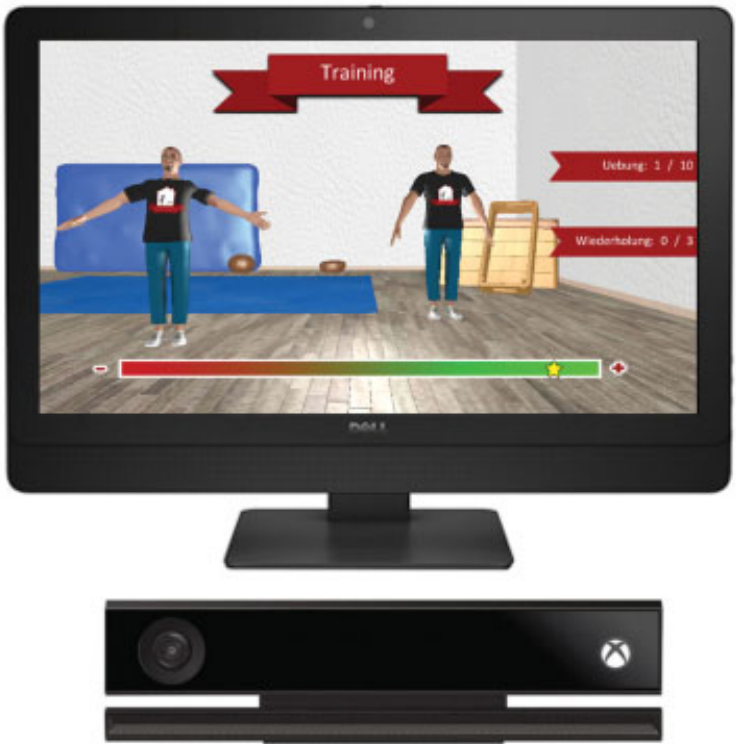

Fig. 1 Training scene in AGT-Reha-V1 (left) and AGT-Reha-V2 (right) in comparison.

based on automatically and securely transmitted exercise data. This comprises only quantity and quality of trainings. Video is neither recorded nor transmitted.

Once the AGT-Reha client software is executed, training starts after a short calibration phase. First, it plays a short video tutorial about the exercise to be performed. Afterwards, it guides the user through the exercise by showing an on-screen three-dimensional (3D) visualization, presenting the intended exercise execution with proper velocity. The depth camera captures the patient's movements and reconstructs a simplified human skeleton represented by a set of joints. Each joint description consists of a 3D position and orientation. These are compared with idealized representations of exercises to estimate and indicate the congruence. The simplified skeleton is mapped to a user avatar (-Fig. 1). That way patients can easily see and correct any deviations in their movements. AGT-Reha-V1 offers a velocity adaptive avatar that moves as slowly as a participant wants to move. The congruence of patient's movements to the idealized exercise is indicated by a traffic light. AGT-Reha-V2 demonstrates the desired movements and velocity by a trainer avatar without adaptions during exercise time. Here, a continuous evaluation of execution is displayed as a colored bar graph. Additionally, too fast or asynchronous movements are indicated by pop up icons. A pause of 30 seconds follows each exercise, as recommended by physical therapists. During this time, a summary of execution quality is displayed and the next exercise gets introduced.

\section{Related Work}

Recent research has shown that depth cameras, such as Microsoft Kinect, Intel RealSense, and Orbecc, are very promising for physical function assessment. ${ }^{16}$ Such marker-less tracking systems are inexpensive and portable, making them more practical than marker-based technologies. Comparisons of the 3D motion analysis systems Microsoft Kinect and Vicon (gold standard), have already demonstrated the potential to be used as reliable and valid clinical measurement tools for recording human motions. ${ }^{17,18}$ The inexpensive Microsoft Kinect cameras have become one of the most used, and also most suited, marker-less tracking systems for telerehabilitation when full body motion is required. ${ }^{19}$ Thereby, tracking of human poses is significantly more robust and accurate with Kinect v2 than with Kinect v1, even though noises can be troublesome, especially at lowers limbs. ${ }^{20,21}$ A few Kinect-based healthenabling technologies supporting patients with MSD in homebased exercises are currently under development. Da Gama et al. developed a prototype to evaluate patient's movement to encourage correct exercising. ${ }^{22}$ Ten participants tested the prototype which can detect abduction and flexion of the shoulder joint by angles. Physical therapists were involved in the development. The prototype correctly recognized all movements classified as wrong or proper. However, the evaluation is limited to abduction and flexion, and does not include rotation. Chung and Chen describe the development process of a mobile game for frozen shoulder rehabilitation to improve the mobility of the shoulder joint in rotation, flexion, extension, abduction, and adduction. ${ }^{23}$ Observations of treatment routines and interviews with patients, physicians, and physical therapists were the basis for the development. So far, only a preliminary evaluation of usability with a group composed of a graphic designer, a therapist, two patients, and three designers was conducted. Anton et al. recently developed and tested a Kinect-based telerehabilitation system for patients with MSD, called KiReS, based on an ontology to select adequate exercises. $^{24}$ The telerehabilitation aspect was covered by a twoway real-time communication. Although the telerehabilitation aspect of KiReS is detailed by means of a fictitious patient, usability and acceptance has only been tested in inpatient settings. In summary, neither this nor any other of the described application systems have been tested without supervision at patients' homes. 
Against this background, the telerehabilitation system called AGT-Reha is presented and evaluated, which was developed in close cooperation with physical therapists to provide automated real-time feedback during the exercises as well as time-delayed feedback from the physical therapists. In contrast to traditional unsupervised home exercise programs, AGT-Reha enables patients to continue their rehabilitation exercises with teletherapeutic support.

\section{Objectives}

The aim of the AGT-Reha-P2 pilot study presented in this article is to evaluate the technical feasibility of the AGT-Reha system at patients' homes. In a more general sense, this study intends to clarify whether health-enabling technology supported home-based rehabilitation is technically feasible and accepted by patients and physical therapists. This requires studying general user acceptance as well as experiences in using AGT-Reha from patients' and physical therapists' perspectives. This also includes processes within the rehabilitation center, which are reviewed to identify adaption requirements for deployment in future routine use.

Furthermore, the study results are used to set up a subsequent clinical effectiveness trial aiming at analyzing the success of medical rehabilitation with respect to the ability to work, capacity to work, and the subjective prognosis of work capacity (e.g., preparing power calculation).

\section{Methods}

The pilot study AGT-Reha-P2 is part of the collaborative research project AGT-Reha by the Peter L. Reichertz Institute for Medical Informatics (PLRI) and the Deutsche Rentenversicherung Reha-Zentrum Bad Pyrmont. AGT-Reha-P2 was conducted as a single-center, nonblinded, nonrandomized, explorative feasibility study comparable to a clinical phase II trial.

\section{Participants}

All participants were consecutively recruited at the Deutsche Rentenversicherung Reha-Zentrum Bad Pyrmont, over a period of 2 years. Each patient participated for 4 months in the study. Given the explorative character of the study no sample size calculation was needed. The main eligibility criteria included patients older than 18 years and inpatient treatment at the rehabilitation center Bad Pyrmont due to a MSD of the shoulder. This includes patients with International Classification of Diseases, Tenth Revision codes of the categories M19 "other arthrosis," especially of the shoulder joint, and M75 "shoulder lesions," for example, frozen shoulder or SIS. Further eligibility criteria were:

- Age between 18 and 65 years.

- Consent to take part in the study.

- Ability to perform the implemented exercises without any health risk.

- Willingness to undertake aftercare with AGT-Reha instead of traditional treatment (medical exercise therapy).
- Place of residence near Braunschweig (30 km) to ensure adequate onsite support.

The main exclusion criteria were patients who were not able to or should not perform the implemented exercises. Furthermore, AGT-Reha had to be able to adequately capture and assess the movements of the patient. Further exclusion criteria were:

- Body mass index $>35$.

- Non-German speakers.

- Pregnancy.

- Participation in another clinical trial, either currently or within the last 30 days.

Checking of inclusion and exclusion criteria, and thus selection of participants, was performed by physicians and physical therapists of the rehabilitation center Bad Pyrmont.

\section{Intervention}

All participants underwent physical training with AGT-Reha over a period of at least 4 months - 1 month within the rehabilitation center, and 3 months aftercare at home. At the beginning of inpatient rehabilitation, participants initially conducted physiotherapeutic self-exercises without AGTReha under supervision of a physical therapist. In doing so, participants learned the various exercises implemented in AGT-Reha as well as their correct execution. After 1 week, each participant was introduced to the training with AGT-Reha from a medical point of view by an attending physical therapist. In addition, participants received a technical briefing on how to assemble and handle the system. At this point, the participants were able to conduct trainings with AGT-Reha in their rooms without therapeutic supervision. Participants could decide how often they engage in training with AGTReha in addition to regular inpatient rehabilitation treatment.

After discharge from rehabilitation center, all participants were asked to train 5 days a week, twice a day for 3 months with AGT-Reha. The duration for each training session was approximately 10 to 15 minutes and included two training sets. A standard training set consisted of nine conventional physiotherapeutic exercises for the shoulder joint, repeated 10 times each.

After each training session, exercise data (quality and quantity) were transferred to the AGT-Reha server. Data screening was done periodically by the physical therapist who treated the patient during their stay at the rehabilitation center. The physical therapists decided whether to contact the patient by telephone, based on their assessment of exercise quality and quantity shown in AGTRehaCare. This served to discuss and adjust incorrectly performed exercises and to motivate patients to train more regularly.

\section{Outcome Measures}

During the trial, participants were assessed by a physician at three measurement points: (T0) prebaseline at admission to rehabilitation center, (T1) baseline at discharge from rehabilitation center, and (T2) posttherapy 3 months after T1 (-Table 1). 
Table 1 Measurement plan-overview of measurement points, parameters, and measuring instruments

\begin{tabular}{|c|c|c|c|c|}
\hline Measuring instrument & Parameter & T0 & T1 & $\mathrm{T} 2$ \\
\hline Neutral-0 method & Active ROM & $\bar{x}$ & $x$ & $x$ \\
\hline Visual analogue scale & Pain & $\mathbf{x}$ & $\mathbf{x}$ & $\mathbf{x}$ \\
\hline AGT-Reha-Acceptance (Q) & User acceptance & & $\mathbf{x}$ & $\mathbf{x}$ \\
\hline Shoulder Pain and Disability Index (Q) & Shoulder function & $\mathbf{x}$ & $\mathbf{x}$ & $\mathbf{x}$ \\
\hline Work Ability Index (Q) & Ability to work & $\mathbf{x}$ & & $x$ \\
\hline Performance Assessment and Capacity Testing (Q) & Capacity to work & $\mathbf{x}$ & & $x$ \\
\hline Subjective prognosis of gainful employment (Q) & Subjective prognosis of work capacity & $x$ & $\mathbf{x}$ & $x$ \\
\hline Semistructured interview & User acceptance, technical feasibility & & & $x$ \\
\hline
\end{tabular}

Abbreviation: Q, questionnaire.

Primary endpoint was the feasibility of exercise training with AGT-Reha at patients' homes. This included technical feasibility and acceptance by practicing patients. While the satisfactory function of AGT-Reha was assessed continuously throughout the entire training period (T0-T2), user acceptance was measured at discharge from rehabilitation center (T1) and posttherapy (T2).

Secondary endpoints were related to the medical rehabilitation success with a view to the estimation of the expected effect size for aftercare with AGT-Reha. Therefore, the active range of motion (aROM) and the shoulder function (pain and disability) were assessed at all three measurement points. In addition, medical rehabilitation success was analyzed with regard to following work-related aspects: (1) ability to work, (2) capacity to work, and (3) subjective prognosis of work capacity. While the subjective prognosis of work capacity was determined at all three measurement points, ability to work and capacity to work were only measured at T0 and T2 due to the length of questionnaires used.

\section{Assessment of Technical Feasibility}

Technical feasibility was measured using a training journal filled out by the participants. Journal items were the date and time of training for each training session, and the overall satisfaction on a five-point scale, from 1 "very satisfied" to 5 "not satisfied." In case of dissatisfaction, the execution of exercises, evaluation of exercises, and technical aspects should have been documented and/or rated in a self-developed questionnaire. Contact with participants due to technical difficulties was also documented to identify and resolve technical errors or general problems as early as possible.

In a semistructured interview at T2 participants had the opportunity to address further technical problems, give suggestions for improvement, and discuss further aspects of training with AGT-Reha.

\section{Acceptance and Usability Evaluation}

User acceptance was measured using a self-developed acceptance questionnaire. The questionnaire consists of 29 items regarding four dimensions: (1) usability, (2) technical satisfaction, (3) acceptance, and (4) medical satisfaction. Each item of the questionnaire could be rated from 0 "apply" to 4 "does not apply." As a complementary measure, the semistructured interview conducted at T2 included questions concerning the motivation, assistance, sustainability, recommendation, and reuse of AGT-Reha. The frequency of use was recorded directly via AGT-Reha exercise data.

\section{Assessment of Shoulder Function}

The aROM was measured using the Neutral-0 Method $^{25}$ (abduction/adduction, flexion/extension, external/internal rotation, and external rotation/internal rotation at 90-degree abduction) by a physician of the rehabilitation center Bad Pyrmont. All measurements of a patient (T0, T1, and T2) were conducted by the same physician to ensure comparability.

Shoulder function (pain and disability) was assessed with the German version of the reliable and valid standardized questionnaire Shoulder Pain and Disability Index (SPADI). ${ }^{26}$ It consists "of 13 items divided into two subscales: pain and disability." ${ }^{21}$ Pain was additionally measured using a visual analogue scale.

\section{Assessment of Work-Related Aspect}

All work-related aspects were measured by different standardized questionnaires. That is, the Work Ability Index ${ }^{27}$ (WAI) to measure ability to work, the Performance Assessment and Capacity Testing ${ }^{28}$ (PACT) to measure capacity to work, and the subjective prognosis of gainful employment ${ }^{29}$ (SPE) to measure the subjective prognosis of work capacity. Furthermore, the final semistructured interview was used to draw conclusions about these aspects.

\section{Statistical Analysis}

Statistical analysis included common descriptive statistical methods, like minimum, maximum, median, as well as relative and absolute frequencies. Evaluating the technical feasibility covers determining absolute frequencies of use of AGT-Reha in days as well as recording and summarizing any emerging problems during the study. Absolute frequencies of answers given in the acceptance questionnaire were combined with statements from the semistructured interviews to derive a general opinion on AGT-Reha from the patients' point of view. In analyzing aROM, in addition to minimum and maximum changes, also the statistical distribution 
was examined to get indications on the effect size of AGT-Reha. Thereby, each movement direction was analyzed individually.

\section{Ethical Considerations}

Ethics approval for this study was granted by the Ethics Committee of Hannover Medical School (ethics approval number: 6558; Ethical Review Board chair: Prof. Dr. Tröger). A written declaration of consent was given by all participants.

\section{Results}

A total of 14 participants aged between 43 and 64 years (mean $=52.25$ ) were enrolled in the trial. Twelve patients with a (chronic) shoulder condition completed the 3 months home-based telerehabilitation exercise program with AGTReha. Two participants were excluded retrospectively as they had received medical exercise therapy in parallel to AGTReha. Consequently, it was not possible to assess their medical rehabilitation success uniquely ascribable to AGTReha.

During the study period, the AGT-Reha system was refined, among other things, due to the market launch of Kinect v2. Therefore, 6 of 12 participants trained with AGT-Reha-V1 (male $=3$, female $=3$ ) and 6 with AGT-Reha-V2 (male $=4$, female $=2$ ). As expected, gender distribution was fairly balanced in both groups ( - Table 2 ).

\section{Technical Feasibility}

Participants were asked to train five times a week for 90 days ( 65 days of training) to maintain and restore mobility. An evaluation of training data showed that all participants trained at least on 45 of the 90 days. One of the participants even trained regularly twice a day. Thus, all participants were able to train on their own with AGT-Reha at home. There were no major technical problems. All minor technical problems as well as difficulties of provisioning at participants' homes were recorded, analyzed, and revised in the system, as far as possible. Some of these problems could be solved directly, others like tracking precision or avatars, were addressed during the development of AGT-Reha-V2. -Table 3 compares the technical problems encountered with AGT-Reha-V1 and AGT-Reha-V2. A clear improvement can be seen, specifically in terms of movement recognition specificity and assessment quality. This positive trend could also be observed in the acceptance questionnaire.

\section{Acceptance}

Almost all participants perceived the system as supportive during their home-based exercise training (11/12). The possibility of self-determined training times, additional motivation, and the perceived security due to external supervision, as well as the fact that the training can be done at home, are some of the most frequently given advantages of aftercare with AGT-Reha as compared with home-based exercises without AGT-Reha or institutional aftercare. All 12 participants would recommend the system, ten would reuse it, and seven indicated their wish to continue the use after their 3month training period. Nine out of the 12 participants stated that AGT-Reha promoted the sustainability of training.

The results of the acceptance questionnaire also clarified these subjective statements regarding acceptance and usability ( - Table 4). The feedback on the following statements was particularly positive: (1) AGT-Reha helps me to remind me of my training, (2) I would recommend this form of aftercare to other patients, (3) and I would choose this aftercare option again. Less well-rated was the statement (5) I trust a computer to evaluate my motions. The opinions were divided regarding the statement "Training with AGTReha makes my shoulder feel better than before." Only a few participants would pay for a permanent use of AGT-Reha and new exercises $($ mean $=1.2)$.

\section{Usability}

Following aspects, mentioned by the participants during the closing interviews, illustrate the usability of AGT-Reha: (1) comprehensible/simple exercises, (2) exercise sessions not too long, (3) easy to use, (4) training is easy to integrate into daily routine, (5) free time allocation/flexibility, and (6) training can be done at home. Aspects influencing the usability negatively, such as "order of exercises always the same" or "time between

Table 2 Participants' characteristics

\begin{tabular}{|l|l|l|}
\hline Characteristics & $\begin{array}{l}\text { AGT-Reha-V1 } \\
(\boldsymbol{n}=\mathbf{6})\end{array}$ & $\begin{array}{l}\text { AGT-Reha-V2 } \\
(\boldsymbol{n}=\mathbf{6})\end{array}$ \\
\hline & Mean \pm SD & Mean \pm SD \\
\hline Age (y) & $47.67 \pm 2.29$ & $56.83 \pm 4.1$ \\
\hline Abduction/adduction (degrees) & $173.33 \pm 39.86$ & $197.5 \pm 37.61$ \\
\hline Flexion/extension (degrees) & $173.33 \pm 26.56$ & $215.83 \pm 7.31$ \\
\hline External/internal rotation (degrees) & $111.67 \pm 17.72$ & $158.33 \pm 8.5$ \\
\hline External/internal rotation at 90 degrees abduction (degrees) & $95 \pm 42.82$ & $142.5 \pm 38.05$ \\
\hline Shoulder Pain and Disability Index (score, 0-130) & $58.67 \pm 15.07$ & $58.16 \pm 18.64$ \\
\hline Work Ability Index (score, 7-49) & $21.33 \pm 7.06$ & $28.83 \pm 9.35$ \\
\hline Subjective prognosis of gainful employment (score, 0-3) & $1.83 \pm 0.69$ & $1.2 \pm 1.17$ \\
\hline
\end{tabular}

Abbreviation: SD, standard deviation. 
Table 3 Technical problems documented by participants during home exercise with AGT-Reha-V1 and AGT-Reha-V2 in comparison (frequency of responses)

\begin{tabular}{|l|l|l|l|}
\hline Category & Problem & AGT-Reha-V1 & AGT-Reha-V2 \\
\hline Patient avatar & Trembling during movements & 6 & 2 \\
\hline & Recognition problems & 2 & 1 \\
\hline Trainer avatar & Avatar hangs in movements & 1 & 0 \\
\hline Exercises and assessment & Bad and incorrect assessment & 6 & 1 \\
\hline & Disturbances & 1 & 0 \\
\hline & Wrong exercise count & 6 \\
\hline Audio and video & Asynchrony of audio and video & 2 & 0 \\
\hline & Hanging videos & 1 & 0 \\
\hline General problems & Software crashes & 1 \\
\hline & System does not run smoothly & 1 & 1 \\
\hline & System change necessary & 2 & 1 \\
\hline & System crash & 1 & 0 \\
\hline & Camera problems & 1 & 0 \\
\hline
\end{tabular}

Table 4 Analysis of AGT-Reha-Acceptance for the dimensions usability and acceptance

\begin{tabular}{|l|l|l|}
\hline Dimension & Statement & Mean (SD) \\
\hline Usability & 1. I think I would like to use this system regularly & $2.75(0.97)$ \\
\hline & 2. I found the system unnecessarily complex & $3.55(1.04)$ \\
\hline & 3. I think the system was easy to use & $3.25(1.54)$ \\
\hline & 4. I think I would need the support of a skilled person to be able to use the system & $3.33(1.37)$ \\
\hline & 5. I found the various functions of the system were well integrated & $2.64(1.63)$ \\
\hline & 6. I think the system is inconsistent & $3.44(1.13)$ \\
\hline & 7. I believe most people would learn to use the system very quickly & $3.42(1.24)$ \\
\hline & 8. I found the system very laborious to use & $3.67(1.15)$ \\
\hline & 9. I felt very safe using the system & $3.67(0.89)$ \\
\hline & 10. I had to learn many things before I could run the system & $4.00(0.0)$ \\
\hline & 1. I'd rather use the system than other forms of rehabilitations & $2.08(0.90)$ \\
\hline & 2. I trust a computer to evaluate my motions & $2.08(1.38)$ \\
\hline & 3. AGT-Reha helps me remember my training & $2.75(1.48)$ \\
\hline & 4. Training with AGT-Reha makes my shoulder feel better than before & $2.33(1.56)$ \\
\hline & 5. I have a problem recording medical data at my home & $2.60(1.65)$ \\
\hline & 6. I would recommend this form of aftercare to other patients & $3.45(1.13)$ \\
\hline
\end{tabular}

Abbreviation: SD, standard deviation.

Note: The valuation was adjusted, a high value being positive for AGT-Reha (scale ranges from 0 to 5 ).

exercises too long" were fixed in the meantime by varying the sequence of exercises and integrating a note on the therapeutic relevance of the pause between two exercises. The video tutorial of a certain exercise is replaced by a simple image instruction, when the performance quality has been above a certain threshold. - Table 4 illustrates the satisfaction regarding the usability of AGT-Reha per statement rated via the AGTReha-Acceptance questionnaire.

\section{Shoulder Function}

The results of aROM measurements showed an average improvement in mobility of the shoulder of at least 8.3 degrees in all movements over the measurement period of 3 months between T1 and T2 (- Fig. 2). The biggest effects could be achieved in internal/external rotation (mean $=10.83$ ) and internal/external rotation at 90-degree abduction (mean $=9.58$ ). Effects could also be seen in terms of adduction/abduction $\quad($ mean $=7.9)$ and flexion/extension $($ mean $=8.3$ ). A detailed visualization of the changes in shoulder mobility (general distribution and changes per participant) can be seen in - Fig. 2. All participants showed an overall improvement in the objectively measured shoulder mobility.

Concerning shoulder function, five of the participants showed a clinically relevant improvement of more than 11 


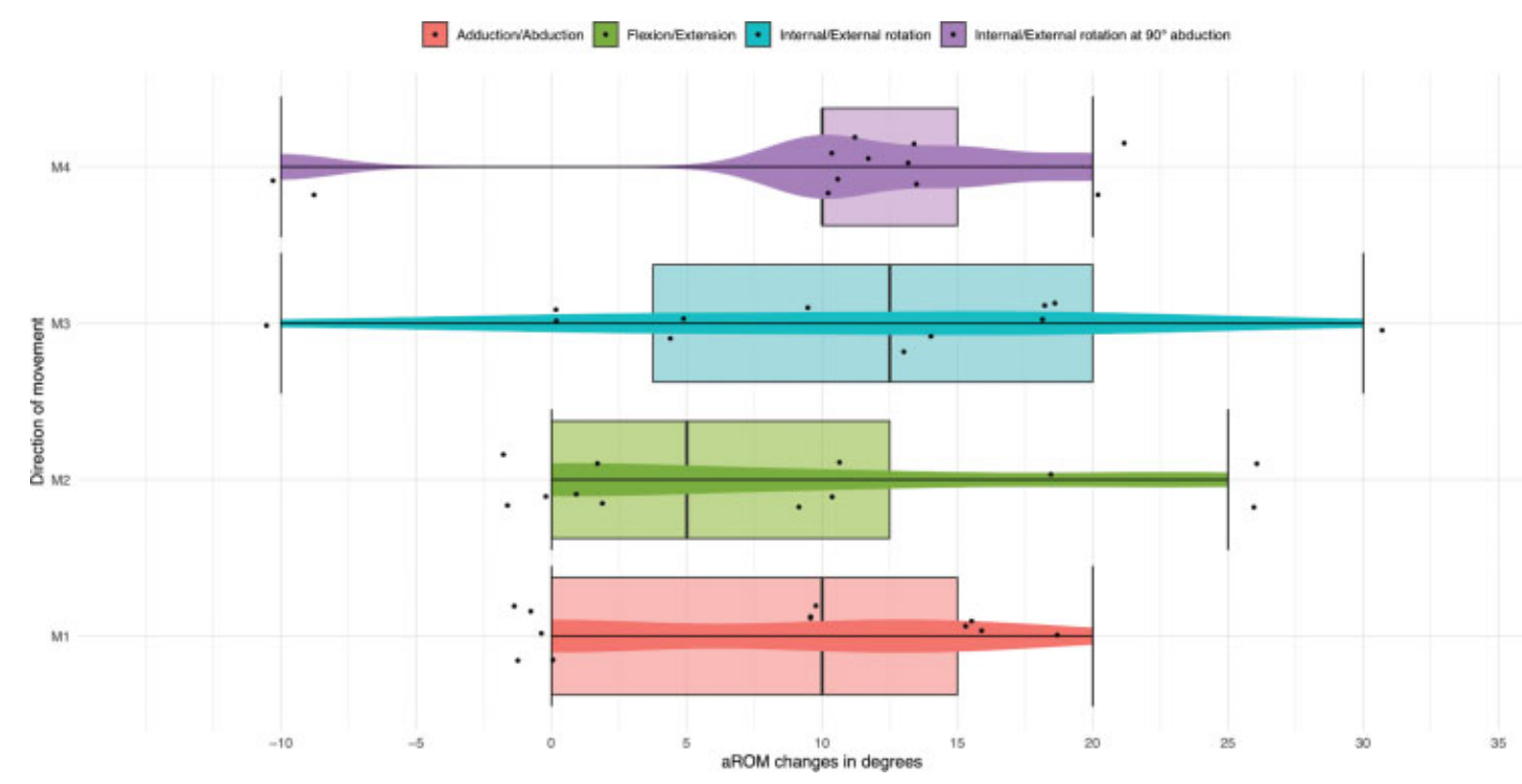

Fig. 2 Boxplot in combination with a violin plot. Changes in shoulder mobility per movement direction in angular degrees (T1-T2).

points in SPADI score. Four participants' scores showed a clinically relevant reduction of shoulder function between $\mathrm{T} 1$ and $\mathrm{T} 2$.

\section{Work-Related Aspects}

An improvement in work ability from T0 to T1 was observed in seven of the 12 participants. In three of these participants this improvement in WAI exceeds $40 \%$. Only one participant showed a noticeable reduction in work ability. In addition to the treated shoulder disease in $\mathrm{T} 2$, this patient also reported suffering from gout. This considerably reduced both the "current ability to work" and the "self-assessment of ability to work during the next 2 years."

The PACT index classifies work capacity as "none," "predominantly in sitting position," "light," "medium," "heavy," and "very heavy" with the possibility of intermediate areas, for example, light to medium. A total of four participants showed an improvement in work capacity from T0 to T2 by at least one class, five remained at the same level, two worsened, and one participant could not be evaluated due to missing values.

\section{Discussion}

Twelve patients with a (chronic) shoulder condition trained for 3 months with AGT-Reha without major technical problems. Regarding the primary endpoint, AGT-Reha can be assessed as technically feasible. The continuity of trainings shows integrability into a broader trial context with a larger number of patients. Minor issues concerning audio and video quality, movement recognition, and assessment were resolved by adjusting the software accordingly (development of AGT-Reha-V2). Just the incorrect count of exercise repetitions could not be solved by this ( - Table 3 ). Therefore, an additional mechanism was implemented which counts the repetitions of the trainer avatar in case of noncounting the patients' exercise repetitions to avoid overstressing of the shoulder.

The surveys showed a very good overall acceptance and high satisfaction in terms of usability among the 12 participants. They would recommend AGT-Reha to other patients. Although some participants reported a need for instructions before running the system initially, they still felt safe using AGT-Reha and assumed that most patients would quickly learn how to use it. This presumably resulted from trial protocol that enforced a careful introduction to the system from just the exercises over training at the clinic to unsupervised training at home.

Participants' willingness to pay for a permanent use of AGT-Reha and new exercises is rather low. This may be explained by the fact that patients do not have to pay for required medical treatments, such as prescribed rehabilitation services, or do only pay copayments in Germany.

Nine patients reported sustainability of the training. This combined valuation supports the several positive observations from the different instruments. Positive effects were also observed with regard to work-related aspects and shoulder function. The aROM measurements showed an average improvement in shoulder mobility with most improvement in internal/external rotation. The changes in the SPADI scores were less obvious. In five out of 12 participants the SPADI score showed a clinically relevant improvement, in four a clinically relevant decline. However, the following interviews suggested that some patients misunderstood the scales of the SPADI questionnaire, as these participants also reported a subjectively perceived improvement in shoulder pain and disability. A logic check confirmed this assumption. For example, "pain perceived as highest" was rated lower within SPADI than the "pain when touching one's own neck." Consequently, the extent to which these results can be interpreted is limited. Further issues were observed in filling out the work-related questionnaires (WAI, PACT, and SPE). Participants had to 
spend a lot of time to complete them caused by their length and complexity. The analyses also revealed a rather low value of SPE and PACT in contrast to the relevance of the WAI in combination with the SPADI.

A variety of subjective questionnaires and forms-standardized or self-developed-were used to evaluate AGTReha. In terms of effectiveness, however, it is obvious to prefer objective measurements. The measurement and documentation of aROM by means of a goniometer and Neutral0 -Method is the clinically established standard. There is, nevertheless, a dependence on the investigator. Furthermore, this method does not allow for continuous measurement of aROM during the entire training period. It would be conceivable to exploit the capabilities of Kinect, that is, the calculation of aROM from positions of joints, to implement a continuous measurement directly in AGT-Reha. There are promising studies on reliability and validity of Kinect for measuring ROM. ${ }^{30,31}$ However, these are strongly linked to the detection accuracy of the camera with all its limitations. Even though this is a possible future component, it has not been implemented yet. Self-administered measurements by patients at their home would at least require a validation study. As the primary endpoints of the presented work are the technical feasibility and user acceptance, the utilized measures focus on these.

One limitation of this study is the small sample size. This allows only a purely descriptive evaluation with tendencies as results. Nevertheless, both the lessons learned and the results of this pilot study were sufficient to refine AGT-Reha, perform a statistical power calculation for a subsequent noninferiority trial, and optimize the study processes. For example, a detailed explanation of questionnaires, in particular of SPADI, is conceivable in a future efficacy trial. Another constraint of AGT-Reha-P2 is related to the ongoing development of the AGT-Reha software as well as the shift from Kinect v1 to Kinect v2. Not only did this make the evaluation of the study more difficult but it also led to delays in the course of the study. However, this procedure also made a patient-centered design possible. Similar to other groups, like Anton et al. ${ }^{24}$ the development and refinement of AGTReha was performed interdisciplinary with medical informaticists, medical rehabilitation experts, physical therapists, and patients.

Given its explorative character, this study cannot assess AGT-Reha's medical effectiveness and its future deployment in routine care. Aspects such as shoulder function and ability to work were assessed and interpreted as secondary endpoints, but could only be partially assessed without valid comparisons to existing methods. For this reason, a clinical trial is planned to show the efficacy and cost-effectiveness of AGT-Reha in comparison to medical exercise therapy. In this way, a decision whether to implement AGT-Reha as an alternative or supplement to existing rehabilitation services can be supported by generating reliable data. Although health-enabling technology-based rehabilitation services do not provide assistance for all patients at any conceivable time in the treatment process, the findings of AGT-Reha-P2 show that such application systems are particularly useful for patients working in shifts or living in rural areas with limited infrastructure. Patients can be enabled to perform their shoulder exercises independently of opening hours of medical centers and physiotherapy practices. This can serve to reduce follow-up costs of nontreatment as well as travel expenses and can save time resources.

In contrast to the presented related work, describing and technically evaluating prototypes, and/or testing usability and feasibility of such systems only in inpatient settings, the results generated by AGT-Reha-P2 suggest the feasibility and acceptance of exercise training with AGT-Reha without supervision at patients' homes. This evaluation is a prerequisite to analyze its efficacy and for future use in patients' homes. Therefore, a first milestone on the way to achieve the aforementioned positive effects of health-enabling technology supported home-based rehabilitation was reached.

\section{Conclusion}

We successfully implemented a health-enabling technology for home-based, technically assisted rehabilitation and evaluated its technical feasibility. The findings of AGTReha-P2 further show that general user acceptance for unsupervised training within AGT-Reha with a group of shoulder patients is given. Supplementary interviews also show the acceptance of physical therapists for teletherapeutic care of patients after discharge from rehabilitation center using the AGTRehaCare Web interface. Furthermore, the hitherto results of SPADI and aROM appear to indicate the effectiveness of aftercare with AGT-Reha. To test this hypothesis, an additional clinical trial with a larger number of participants needs to be conducted. With some caution, the results can be transferred to other MSD or even other indications. The results of this study indicate that healthenabling technology supported home-based exercise programs are feasible.

Future work will utilize the refinement of AGT-Reha-V2 based on the participants' feedback. For example, instead of an avatar the patient's mirror image will be displayed to improve the movement feedback, and thus, increase patients' motor (re-)learning. Adding more exercises, also for other indications, could further increase user acceptance and effectiveness, and thus facilitate the integration into health care processes. Apart from that, it is conceivable to include further applications, for example, simple smartphone-based exercise instructions for training away from home, information and knowledge about the disease and therapy, as well as self-assessment possibilities. ${ }^{32,33}$ Furthermore, the evaluation of AGT-Reha is currently running using a noninferiority trial to compare the efficacy of AGT-Reha with medical exercise therapy (DRKS-ID: DRKS00011596).

\section{Funding}

The AGT-Reha project is funded by the Deutsche Rentenversicherung Braunschweig-Hannover, Germany. Open Access funding is provided by the German Research Foundation and the Open Access Publication Funds of the Technische Universität Braunschweig. 


\section{Conflict of Interest}

This work is part of the collaborative research project AGT-Reha funded by the Deutsche Rentenversicherung Braunschweig-Hannover. They provided hardware and support for the study described in the manuscript. There are no other individual conflicts of interest.

\section{Acknowledgments}

The authors would like to thank all involved in the AGTReha project for their assistance while planning and performing the study: Stefan Franz for developing the initial AGT-Reha version 0.9, Julius Saretzki for advancement of AGT-Reha to version 1.9, Jan Pollack as the main developer of AGTRehaCare, Monika Schwarze for assisting in study planning, Jana Riquel and Christina Eberth for supervision of the study participants, and Nils Backenköhler, Phil Lindow and Lennart Drescher for their valuable contributions. Special thanks is due to the physical therapists of the rehabilitation center Bad Pyrmont for their support in developing AGT-Reha as well as taking over therapeutic support of participants during their home-based exercise program with AGT-Reha.

\section{References}

1 Woolf AD, Erwin J, March L. The need to address the burden of musculoskeletal conditions. Best Pract Res Clin Rheumatol 2012; 26(02):183-224

2 Robert Koch-Institut. Gesundheit in Deutschland. Gesundheitsberichterstattung des Bundes. Gemeinsam getragen von RKI und Destatis. Berlin: H.Heenemann GmbH \& Co. KG; 2015 [German]

3 BARMER GEK. Gesundheitsreport 2014 Niedersachsen. Berlin: BARMER GEK; 2014:8-75 [German]

4 Mitchell C, Adebajo A, Hay E, Carr A. Shoulder pain: diagnosis and management in primary care. BMJ 2005;331(7525):1124-1128

5 Deutsche Rentenversicherung. Reha-Bericht 2018: Die medizinische und berufliche Rehabilitation der Rentenversicherung im Licht der Statistik. Berlin: Deutsche Rentenversicherung Bund; 2018 [German]

6 World Health Organization. WHO Guidelines on Health-Related Rehabilitation (Rehabilitation Guidelines). 2014 Available at: https://www.who.int/disabilities/care/rehabilitation_guidelines_concept.pdf. Accessed October 18, 2019

7 Bundesarbeitsgemeinschaft für Rehabilitation. Praxisleitfaden: Strategien zur Sicherung der Nachhaltigkeit von Leistungen zur medizinischen Rehabilitation. Frankfurt/Main: BAR; 2016 [German]

8 Haux R, Koch S, Lovell NH, Marschollek M, Nakashima N, Wolf K-H. Health-enabling and ambient assistive technologies: past, present, future. Yearb Med Inform 2016(Suppl 1):S76-S91

9 Evolv Rehabilitation Technologies. S.L. Virtual Rehab. 2019. Available at: https://evolvrehab.com/virtualrehab/. Accessed September 29, 2019

10 Wang Q, Markopoulos P, Yu B, Chen W, Timmermans A. Interactive wearable systems for upper body rehabilitation: a systematic review. J Neuroeng Rehabil 2017;14(01):20

11 Dimaguila GL, Gray K, Merolli M. Person-generated health data in simulated rehabilitation using Kinect for stroke: literature review. JMIR Rehabil Assist Technol 2018;5(01):e11

12 Bonnechère B, Jansen B, Omelina L, Van Sint Jan S. The use of commercial video games in rehabilitation: a systematic review. Int J Rehabil Res 2016;39(04):277-290

13 Peretti A, Amenta F, Tayebati SK, Nittari G, Mahdi SS. Telerehabilitation: review of the state-of-the-art and areas of application. JMIR Rehabil Assist Technol 2017;4(02):e7
14 EvoCare Holding. Health is coming home. 2019. Available at: https://telemedizin.de/english/home. Accessed October 17, 2019

15 Wolf KH, Franz S, Schwartze J, et al. "AGT-Reha": Assistierende Gesundheitstechnologien für das medizinische Tele-Reha-Training. Abstract presented at: 58th Annual Conference of the German Association for Medical Informatics, Biometry and Epidemiology (GMDS); September 01-05, 2013; Lübeck, Germany [German]

16 Clark RA, Mentiplay BF, Hough E, Pua YH. Three-dimensional cameras and skeleton pose tracking for physical function assessment: a review of uses, validity, current developments and Kinect alternatives. Gait Posture 2019;68:193-200

17 Galna B, Barry G, Jackson D, Mhiripiri D, Olivier P, Rochester L. Accuracy of the Microsoft Kinect sensor for measuring movement in people with Parkinson's disease. Gait Posture 2014;39(04): 1062-1068

18 Otte K, Kayser B, Mansow-Model S, et al. Accuracy and reliability of the Kinect Version 2 for clinical measurement of motor function. PLoS One 2016;11(11):e0166532

19 Mousavi Hondori H, Khademi M. A review on technical and clinical impact of Microsoft Kinect on physical therapy and rehabilitation. J Med Eng 2014;2014:846514

20 Mortazavi F, Nadian-Ghomsheh A. Stability of Kinect for range of motion analysis in static stretching exercises. PLoS One 2018;13 (07):e0200992

21 Wang Q, Kurillo G, Ofli F, Bajcsy R. Evaluation of Pose Tracking Accuracy in the First and Second Generations of Microsoft Kinect. Paper presented at: International Conference on Healthcare Informatics; October 21-23, 2015; Dallas, TX, USA

22 Da Gama A, Chaves T, Figueiredo L, Teichrieb V. Guidance and Movement Correction Based on Therapeutics Movements for Motor Rehabilitation Support Systems. Paper presented at: 14th Symposium on Virtual and Augmented Reality; September 10, 2012; Rio de Janiero, Brazil

23 Chung C-E, Chen C-H. The app game interface design for frozen shoulder rehabilitation. In: Soares M, Falcão C, Ahram T, eds. Advances in Ergonomics Modeling, Usability \& Special Populations. Cham: Springer; 2017:507-516

24 Anton D, Berges I, Bermúdez J, Goñi A, Illarramendi A. A telerehabilitation system for the selection, evaluation and remote management of therapies. Sensors (Basel) 2018;18(05):1459

25 Castro WHM, Jerosch J. Examination and Diagnosis of Musculoskeletal Disorders: History - Physical Examination - Imaging Techniques - Arthroscopy. New York: Thieme; 2011

26 Breckenridge JD, McAuley JH. Shoulder Pain and Disability Index (SPADI). J Physiother 2011;57(03):197

27 Tuomi K, Ilmarinen J, Jahkola A, Katajarinne L, Tulkki A. Work Ability Index. 2nd ed. Helsinki: Institute of Occupational Health; 1998

28 Gibson L, Strong J. The reliability and validity of a measure of perceived functional capacity for work in chronic back pain. J Occup Rehabil 1996;6(03):159-175

29 Mittag O, Raspe $\mathrm{H}$. A brief scale for measuring subjective prognosis of gainful employment: findings of a study of 4279 statutory pension insurees concerning reliability (Guttman scaling) and validity of the scale [in German]. Rehabilitation (Stuttg) 2003;42(03):169-174

30 Saalfeld B, Pingel I, Wolf KH. Semi-automatically measuring shoulders' range of motion - objective measurements with good reliability and accuracy. Stud Health Technol Inform 2018;247:631-635

31 Çubukçu B, Yüzgeç U, Zileli R, Zileli A. Reliability and validity analyzes of Kinect V2 based measurement system for shoulder motions. Med Eng Phys 2020;76:20-31

32 Steiner B, Wolf KH. A vision utilizing gamification to enhance patients' with chronic shoulder diseases adherence to rehabilitation. Stud Health Technol Inform 2019;262:71-74

33 Steiner B, Wolf KH. A Mobile Application Enhancing Adherence to Rehabilitation: A Vision. Abstract presented at: 64th Annual Conference of the German Association for Medical Informatics, Biometry and Epidemiology (GMDS); September 08-11, 2019; Dortmund, Germany 